\title{
SUR UNE NOUVELLE ESPÈCE DE STREPTOMYCES
}

\author{
(STREPTOMYCES GALTIERI n. sp.), ISOLÉE
}

\section{D'UN CAS D'ACTINOMYCOSE SEPTICÉMIQUE CHEZ LE CHIEN}

\author{
Par P. GORET et L. JOUBERT
}

Au cours de l'année 1948, nous avons eu l'occasion de déceler chez un chien de trois mois une "actinomycose " septicémique, dont nous avons donné ailleurs (1) la relation. Du point de vue clinique, nous soulignions alors l'intérêt de cette observation dans les termes suivants :

« On peut conclure :

" $1^{\circ}$ Qu'il s'agit d'un cas d' " actiromycose » généralisée et septicé« mique du chien. En effet, l'ubiquité des lésions thoraciques, abdomina* les et cérébrales et du germe spécifique ne peut laisser de doute sur ce « sujet.

«2॰ Qu'il s'agit d'un processus rapide puisque, en accord avec les 《 commémoratifs, les « grains 》 actinomycosiques, si caractéristiques * de la longueur d'évolution de cette infection, ne purent jamais être « décelés dans le cas présent. La réaction vasculo-sanguine, l'intégrité

* des tubes urinifères, la présence, au sein de la moelle osseuse elle« même, d'éléments en multiplication active (croissance angulaire \& d'ørskov), confirment ce caractère.

" $3^{\circ}$ Qu'on est donc bien en présence d'une forme non encore décrite « d' « actinomycose » canine, réunissant, en un seul et même processus « morbide d'évolution rapide, des formes cliniques considérées comme « rares ou exceptionnelles chez le chien (actinomycoses nerveuses et « viscérales). »

Il nous paraît intéressant de rendre compte de l'étude détaillée du germe en cause, déjà amorcée dans notre précédente publication.

Le germe responsable, aérobie strict, présente des caractères qui le différencient nettement des Actinomycétales pathogènes jusqu'alors décrites.

(1) P. Gonet et L. Joubert. - Rev. Méd. Vét., C, 1949, 561 .

Ann, de Parasitologie, T, XXVI, Nos $1-2, \ldots 1951$, 


\section{I. - CARACTERES MORPHOLOGIQUES}

1. Bouillon et gélose nutritive ordinaires, - Ce germe n'échappe pas à la règle qui confère aux Actinomyces un très grand polymorphisme.

A l'état jeune, il forme de longs filaments flexueux, de 3 à $25 \mu$ de long sur $1 \mu$ de large, donnant des ramifications généralement perpendiculaires au filament principal, uniques au point d'implantation. Parfois, certains éléments bacilliformes s'associent par deux

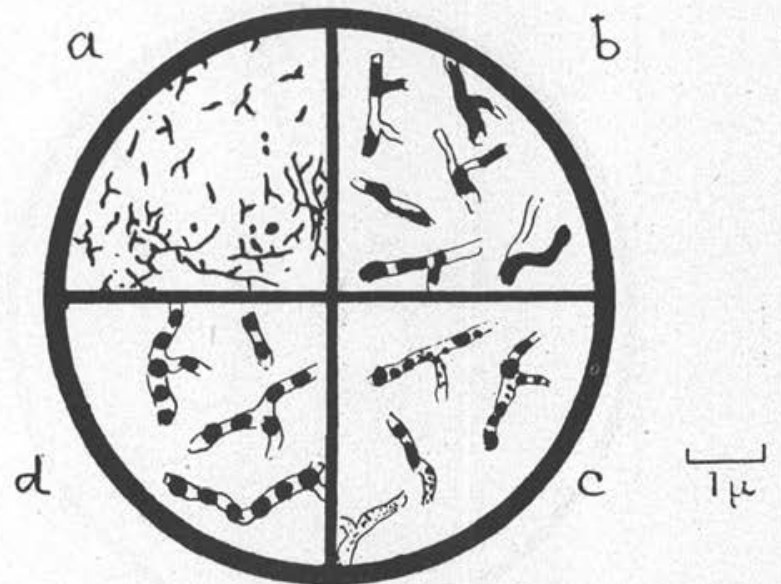

Fig. 1. - Représentation demi-schématique de l'aspect microscopique de l'Actinomyces et de son mode de fragmentation. a) Frottis sur une culture jeune.

b) Condensation pıotoplasmique irrégulière. c) Granulisation. d) Endospores.

ou trois et présentent des figures alphabétiques en $\mathrm{X}, \mathrm{Y}$ ou L (croix d'ørskov).

On n'observe ni flagelles, ni capsules. Caractère important: il n'existe pas de spores latérales pédicellées (fig. 1, a).

Ces éléments allongés ne tardent pas, en culture surtout, à se fragmenter selon des modes multiples qu'il faut préciser, en raison de leur importance pour la classification du germe :

a) Le protoplasma se concentre en certains points, de manière irrégulière, et les ponts filamenteux qui les joignent se lysent, laissant en place des éléments de toutes formes et de toutes dimensions (sphérules, ovoïdes, formes en $\mathrm{S}$, en $\mathrm{U}$, quelquefois même streptobacilles) (fig. 1, $b$ ). 
b) Un mode spécial de granulisation peut engendrer des éléments arrondis de toutes grosseurs, mais d'un diamètre toujours inférieur au filament initial ; cette poussière protoplasmique peut parvenir jusqu'à la limite de la visibilité (fig. $1, c$ ). Ces types de fragmentatiı n, absolument banals chez les Actinomycètes, n'offrent que peu d'intérêt.

c) -Il n'en est pas de même pour un autre mode de fragmentation, qui consiste dans la condensation protoplasmique régulière des éléments actinomycosiques. Les formations ovalaires qui en résultent,

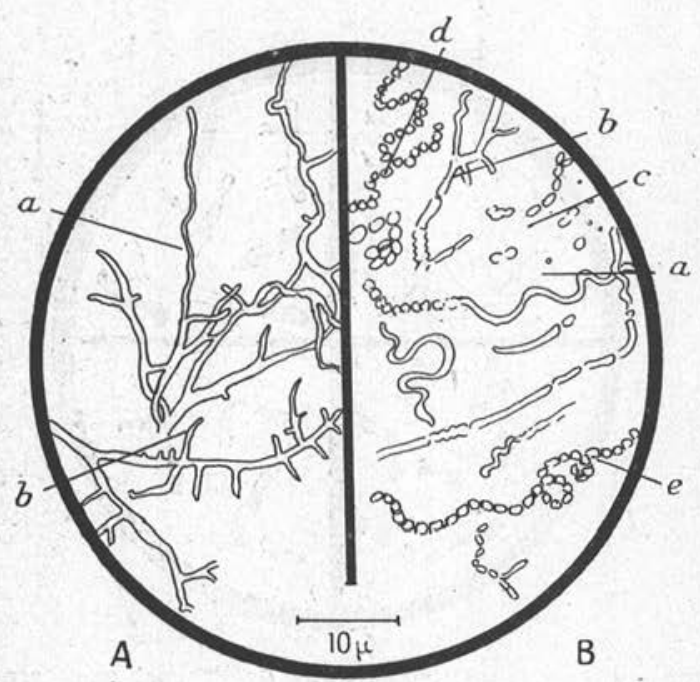

Fig. 2. - Aspects en MilieuX sucrés. A.. Gzapek glycosé de Waksman. - (Après 15 jours d'étuve à $25^{\circ}$ C.). a) Aspect banal dans l'épaisseur du milieu. b) Morphologie du mycélium rampant sur la paroi de verre. B. Gélose glycosée de Sabouraud. - (Après 5 jours d'étuve à $30^{\circ}$ C.). a) Filaments allongés. $b$ ) Condensations protoplasmiques irrégulières. $c$ ) Ebauche de granulisation. d) Endospores. e) Endospores et vrilles.

ou « endospores », constituent l'efflorescence, très caractéristique des cultures d'un groupe particulier d'Actinomycétales : les Streptomyces (fig. 1, $d$ ).

2. En milieux sucrés, - Filaments rampant sur milieux solides ou filaments immergés dans les milieux liquides. - Sur les parois de verre, filaments flexueux, avec ramifications le plus souvent à angle droit. Le diamètre est de $0 \mu, 3$ à $0 \mu, 8$ (fig. $2, \mathrm{~A}, a$ et $b$ ).

Filaments aériens, - Ramifiés, formant des vrilles sinistrorses, 
ANNALES DE PARASITOLOGIE

T. XXVI, Nos 1-2, 1951.
Planche I

Mémoire P. Goret et L. Joubert.

FIG, 3.- Sur bouillon (les tubes de culture ont été inclinés) : $a$, voile; $b$, colonie isoléé.

Fig. 4. - Sur gẻlose: $a$, nappe; $b$, colonies isolées (on distingue la dissociation en colonies $A$ et $B$, ces dernières se massant, de préférence, dans le fond du tube). 


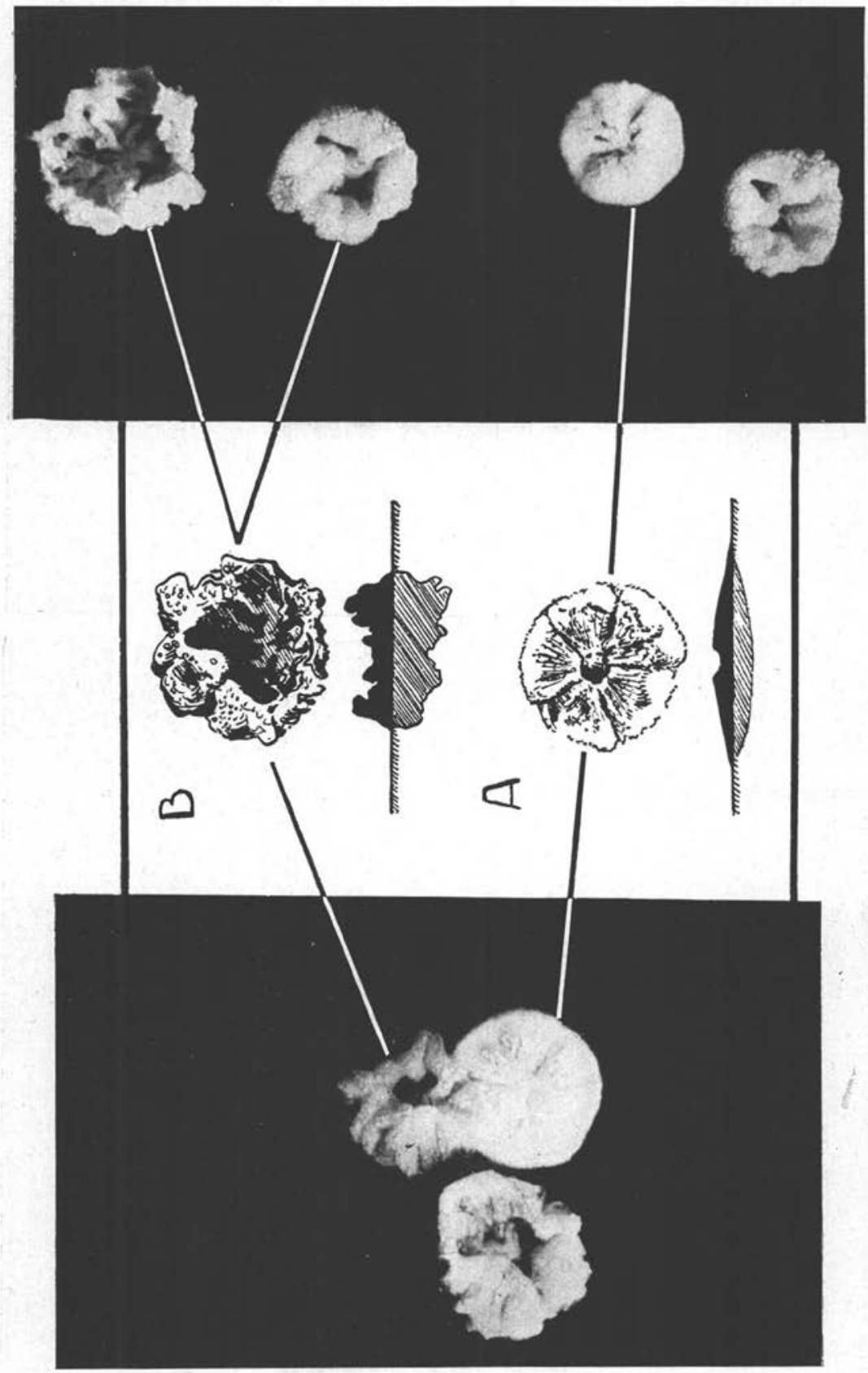

FIg. 5 et 6 . - Dissociation en milieu solide (plan et coupe) Masson et $\mathrm{C}^{10}$, Editeurs 
se fragmentant en arthrospores rondes ou en bâtonnets, qui peuvent présenter des figures en $\mathrm{X}$ ou en $\mathrm{Y}$, lesquelles, plus tard, se fragmentent également en conidies. Diamètre $\pm 0 \mu, 8$.

Endospores ou « conidies ». - Ovales, 0,8-1 $\mu, 5 \times 0 \mu, 8$ (fig. 2 , $\mathrm{B}, d$ et $e$ ).

\section{II. - COLORATION}

Ce germe se colore très facilement par les colorants d'aniline et, en particulier, garde le Gram. Aucune forme acido-résistante ne peut être décelée.

\section{III. - CARACTERES CULTURAUX}

\section{Milieux nutritifs ordinaires}

Les cultures se réalisent très facilement en aérobiose à une température voisine de $37^{\circ}$, dans les milieux usuels de laboratoire. Les exigences respiratoires et thermiques de ce germe sont assez strictes à l'isolement. Cependant, une atmosphère enrichie en $\mathrm{CO}^{2}(10$ à 20 p. 100) s'est révélée particulièrement favorable à sa croissance.

En milieu liquide, les colonies - ou plus exactement les thalles - forment de petits flocons qui se déposent au fond du tube. Ils constituent, en surface, un voile blanc, sec, écailleux, fragile, tendant à remonter le long de la paroi de verre (fig. $3, a$ ). Le milieu ne se trouble jamais.

La colonie isolée en voile, sur bouillon, prend un aspect cratériforme, comparable à celui des colonies isolées sur gélose (fig. $3, b$ ).

Sur milieu solide, apparaissent, en trois à quatre jours, des thalles de la grosseur d'une tête d'épingle, hémisphériques, d'un blanc de plâtre, secs, écailleux, inodores. En certains milieux (pomme de terre notamment), un pigment discret, jaune rosé, se développe. En quelques jours, les colonies, confluentes lorsque l'ensemencement a été suffisamment abondant, donnent l'apparence d'une nappe plâtreuse et rugueuse (fig. $4, a$ ). Les colonies isolées, en revanche, doublent et triplent de volume et s'ombiliquent rapidement, sans jamais tendre vers la confluence (fig. $4, b$ ). Après vieillissement de la colonie, apparaît une coloration brune, qui ne correspond pas à un véritable pigment, mais à la production d'une quinone oxydable en aérobiose.

Dissociation. - Au cours de cette étude bactériologique, se révéla, sur milieux solides, un curieux phénomène de dualité mor- 
phologique dans les colonies baptisées arbitrairement «A $\mathrm{A}$ et «B $\mathrm{B}$ (voir fig. 5 et 6 ). En voici succinctement le détail :

Colonie «A». - Colonie petite, plate, ombiliquée, blanche, enchâssée dans la gélose en cupule régulière, à surface finement granuleuse, striée radiairement et comme givrée, donnant en bouillon un voile pelliculaire fragmenté en larges plaques.

Colonie « $B$ ». - Colonie grande, surplombant fortement la surface de la gélose, donnant l'apparence d'une éponge, de couleur chamois, encastrée dans la gélose profondément et irrégulièrement, à surface lisse, parsemée de minuscules gouttelettes de sérosité, donnant en bouillon un voile granulaire difficilement dissociable.

Les caractères morphologiques particuliers à chacun des types de colonies se transmettent régulièrement par repiquage en série sur gélose nutritive ordinaire.

Comme les deux types ne se différencient, en dehors des caractères morphologiques, que par un comportement différent de la culture en $\mathrm{CO}^{2}$ d'une part (colonie $\mathrm{B}=$ culture en atmosphère de $\mathrm{CO}^{2}$; colonie $\mathrm{A}=$ pas de culture en atmosphère de $\mathrm{CO}^{2}$ ) et de l'utilisation du citrate de soude d'autre part (colonie $\mathrm{B}=$ citrate + ; colonie $\mathrm{A}=$ citrate - ), nous pensons qu'il s'agit bien d'un phénomène de dissociation, encore inédit chez les Actinomycétales.

\section{2. - Milieux spéciaux *}

Czapek saccharesé gélosé selon Waksman (2). -8 jours, $p \mathrm{H} \mathrm{6,1.}$

Thalles restreints couverts d'une efflorescence blanche, farineuse. Pas de pigment soluble.

Czapek glycosé gélosé selon Waksman (2). -8 jours, $p \mathrm{H}$ 6,1.

Croissance un peu plus forte que sur Czapek saccharosé. Pas d'autres différences.

Gélose au malate de calcium (5). - 14 jours, $p \mathrm{H} 7$.

Thalles restreints, granuleux, couverts d'une efflorescence blanche, farineuse. Les revers (***) sont de couleur rose-orangée (CC. 128 D103) (**). Pas de pigment soluble.

(*) Observations faites sur cultures à la température de $25^{\circ}$, sauf indication contraire.

Le chiffre () suivant l'indication de chaque milieu correspond à celui numérotant les milieux dans : Waksmas, Cultural studies of species of Actinomyces. Soil. Sc., VIII, 1919, p. 81-83.

(**) Les symboles utilisés pour exprimer la coloration sont tirés de P. Klincksieck et Th. Valette, Code des Couleurs, Paris, 1908.

$(* * *)$ Le revers de la colonie correspond à la partie visible par examen à travers le milieu-support, 
Milieu amidonné de Waksman (15). - 14 jours, $p H$ 7,2.

Thalles restreints, minces, couverts d'efflorescence blanche, farineuse, à bords lobés irrégulièrement. Pas de pigment soluble.

Pomme de terre glycérinée, $\mathrm{pH} 6,6$. - 14 jours.

Thalles punctiformes; forment en confluant une croûte épaisse, granuleuse de couleur orange-rosée $(\mathrm{CC}$. $78 \mathrm{C}-103 \mathrm{C}$ ). Les revers sont très ondulés et de la même couleur. Efflorescence apparaissant très tard, blanche, fine, toujours limitée. La pomme de terre n'est pas pigmentée. Pas de pigment soluble.

Gélatine (Goldlabel) (18). -8 jours, $\mathrm{pH} 5,7,20^{\circ} \mathrm{C}$.

Thalles nuls ou très petits, punctiformes, couverts d'une efflorescence blanche, farineuse. Liquéfaction très limitée : petit bouton de liquéfaction autour du point d'ensemencement.

Pomme de terre gélosée. - 8 jours, $p \mathrm{H} 6,6,20^{\circ} \mathrm{C}$.

Thalles restreints, couverts d'une efflorescence blanche, farineuse, avec quelques fentes concentriques. Les revers sont de couleur orange-rosée (CC 128 D). Pas de pigment soluble.

Gélose nutritive de Waksman (8). - 14 jours, $p \mathrm{H} \mathrm{5,9.}$

Thalles restreints, minces, plats, couverts d'une efflorescence blanche, farineuse, à bords arborisés. Les revers sont d'une couleur jaunâtre (CC152). Pigment soluble brun clair.

Gélose nutritive glycérinée de Waksman (8). - 14 jours, $p \mathrm{H}$ 5,9.

Développement meilleur que sur le milieu précédent. Thalles punctiformes, formant une croûte granuleuse. Efflorescence blanche, fine. Les revers sont orangés-jaunâtres (CC 156). Pigment soluble peu intense, brun clair.

Gélose saccharosée de Sabouraud. - 14 jours, $p H$ 5,7.

Bonne croissance. Les colonies confluentes forment une nappe plâtreuse, rugueuse, blanche, très légèrement rosâtre. Les revers sont orangés (CC 137-127). Pas de pigment soluble.

Gélose peptonée. - 14 jours, $\mathrm{pH} 5,3$.

Thalles très restreints, couverts d'une efflorescence blanche, farineuse. Les revers sont crémeux. Pigment soluble très peu intense, brun roussâtre.

Milieu glucosé peptoné de Waksman (9). - 14 jours, $p H 7$.

Croissance abondante. Nombreux thalles punctiformes formant un voile à la surface, couvert d'une efflorescence. Dans le liquide : grands flocons hyalins. Pigment soluble peu intense, brunâtre. .

Czapek amidonné (1). - 14 jours, $\mathrm{pH} 7,5$.

Thalles formant un voile extrêmement mince, hyalin en surface. Pas d'efllorescence. Dans le liquide, quelques flocons hyalins. Fas de pigment soluble. 
Czapek saccharosé (1). - 14 jours, $\mathrm{pH} 7,5$.

Même aspect que sur Czapek amidonné.

Czapek glycériné. - 14 jours, $p \mathrm{H} 7,5$.

Thalles punctiformes, formant un voile épəis, couverts d'une efflorescence. Dans le liquide, quelques flocons hyalins. Pas de pigment soluble.

Lait écrémé (17). - \pm 14 jours, $p \mathrm{H} 6$.

$25^{\circ} \mathrm{C}$. Croissance assez lente. Thalles formant un voile mince, couverts d'une efflorescence blanche. Les thalles fixés aux parois du verre ont un revers jaune-orangé (CC 151). Pas de pigment soluble.

$37^{\circ} \mathrm{C}$. Pas de diffèrences avec les cultures à $25^{\circ} \mathrm{C}$.

Sérum de bœuf gélosé. - 16 jours, $\mathrm{pH} 7,0$.

Thalles plats, humides, blancs-grisâtres. Efflorescence très mince. Milieu se décolorant de blanc à brun violet (CC 122) au-dessous des thalles.

Gélose au sang. - 16 jours, $p H 7,0$.

Thalles restreints, un peu surélevès, couverts d'une efflorescence se fendillant dans les parties les plus élevées.

Milieu à l'œuf (de Lœwenstein), rouge. - 16 jours, $p H$ 7,0.

Thalles formant une couche surélevée, granuleuse, blanche grisâtre, presque entièrement couverte d'efflorescence. Milieu ne se décolore pas.

Milieu à l'œuf (de Lœwenstein), vert. - 15 jours, $p \mathrm{H} \mathrm{7,0.}$

Comme sur le milieu précédent, mais efflorescence moins abondante et très mince. Milieu se décolore du vert au jaune.

\section{IV. - CARACTERES BIOCHIMIQUES}

a) Action sur les glucides. - Tant en milieu de Hiss qu'en eau peptonée additionnée d'un indicateur de $p \mathrm{H}$, aucun virage n'a été décelé vis-à-vis des alcools, oses, polysaccharides ou glucosides usuels, même après 20 jours d'étuve à $37^{\circ}$.

Cependant, l'inversion du saccharose est positive après 14 jours d'étuve (Czapek saccharosé).

b) Action sur les protides. - Aucune action sur le sérum coagulé ; liquéfaction très faible de la gélatine.

c) Produits formés dans les milieux de cultures :

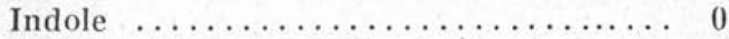

Acétyl-méthyl-carbinol $\ldots \ldots \ldots \ldots \ldots, 0$ 
d) Réduction des nitrates en nitrites :

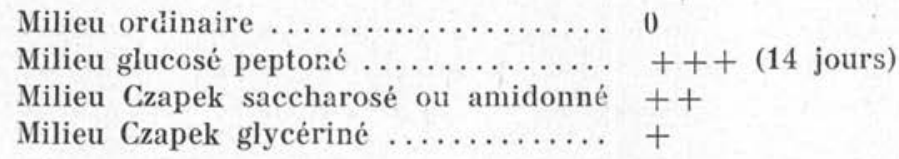

e) Changement de réaction (14 jours). - Inchangée dans le lait à $25^{\circ}$ ou $37^{\circ} \mathrm{C}$. ; légèrement acide dans le milieu de Czapek saccharosé ou amidonné; nettement alcalin quand ce dernier milieu est glycériné.

f) Action diastasique (14 jours). - Négative.

g) Action sur le lait écrémé. $-25^{\circ} \mathrm{C}$., nulle ; $37^{\circ} \mathrm{C}$., coagulation de lait après environ 20 jours. Pas de digestion du caillot.

\section{V. - CARACTERES BIOLOGIQUES}

Pouvoir antibiotique. - Aucune sécrétion antibiotique n'a pu être décelée vis-à-vis des bactéries Gram positives (staphylocoque) et Gram négatives (colibacille).

Vitalité et résistance du germe. - Assez fragile, il se conserve environ deux mois en gélatine, à la température du laboratoire.

\section{VI. - POUVOIR PATHOGENE EXPERIMENTAL}

Le cobaye est sensible à l'inoculation intra-péritonéale de $0,3 \mathrm{cc}$. de la suspension homogène (broyage au mortier) d'un mélange de culture en bouillon et gélose de 72 à 96 heures.

Il succombe en quatre à huit jours avec des lésions de péritonite exsudative. On note la présence de fausses membranes fibrineuses et de nodules au niveau des différents organes splanchniques. Les lésions sont riches en germes.

L'ensemencement du liquide péritonéal permet d'obtenir une culture pure du germe.

Le lapin s'est montré sensible à l'inoculation intra-veineuse de $1 \mathrm{cc}$. de suspension. Il succombe en 72 à 96 heures.

Le chien s'est montré insensible à l'inoculation intra-veineuse et intrapéritonéale de $3 \mathrm{cc}$. d'une suspension épaisse de germes. Ce fait ne laisse pas d'être paradoxal, puisque à l'origine le germe a été isolé chez le chien. 
Le pouvoir pathogène accuse une différence profonde entre les deux variantes ; seules, les cultures obtenues à partir de A se montrent pathogènes pour le cobaye, celles issues de B n'étant pourvues que d'une virulence fort discrète envers cet animal. Dans les deux cas, on réisole des lésions un mélange A-B, dont les caractères se transmettent en série.

\section{VII. - DETERMINATION ET PLACE}

\section{DANS LA. SYSTEMATIQUE}

Le germe qui fait l'objet de notre relation apparaît difficile à déterminer.

Nous référant à la classification de Waksmann et Henrici, utilisée par Bergey ( $6^{\circ}$ édition, 1948), il ne fait aucun doute que nous avons affaire à un germie de la classe des Schizomycètes, ordre des Actinomycétales. La famille est plus difficile à préciser.

Ce n'est pas un Actinomyces, puisqu'il est aérobie.

Ce n'est pas un Micromonospora, puisqu'il n'a pas les spores terminales isolées, pédicellées et qu'il n'est pas fortement thermophile.

En revanche, il présente des caractères se rapportant soit à Streptomyces, soit à Nocardia.

En effet, c'est un Streptomyces : par la présence d'efflorescence (mycélium aérien visible en culture sur lame) ; par son appareil sporifère spiralé ; par sa culture sur pomme de terre.

Toutefois, il s'écarte du genre Streptomyces: parce qu'il ne dégage pas d'odeur ; il ne liquéfie pas le sérum coagulé ; il ne liquéfie que faiblement la gélatine ; enfin, parce qu'il possède un pouvoir pathogène relativement élevé, fait rare chez les Streptomyces.

Les caractères qui le rapprochent de Nocardia sont surtout la fragmentation du mycélium et son pouvoir pathogène, joints précisément aux caractères indécis qui le séparent des Streptomyces.

Pour nous, ces caractères sont insuffisants pour éliminer ce germe de la famille des Streptomycétacées et nous pensons, au contraire, pouvoir le ranger à côté des quelques Streptomyces faiblement protéolytiques ( $S$. fordii, africanus, galliens, pelletieri, cisteri, upcotti, hortonensis), mais tous pathogènes pour l'homme, et créer une nouvelle espèce. Nous proposons le binôme Streptomyces galtieri, en hommage au Professeur Galtier, premier titulaire de la chaire de bactériologie à l'Ecole Vétérinaire de Lyon et premier Chef du Service où ce germe fut récemment isolé. 
Nous avions, un moment, pensé faire de ce germe une souche aberrante pathogène d'Actinomyces carneus (Rossi doria) et créer une nouvelle combinaison : Streptomyces carneus, étant donné les ressemblances existant entre notre germe et les souches étudiées par comparaison : Cl. et B. 1.602 de Baldacci.

Mais, d'une part, les caractères d'A. carneus et sa classification sont encore mal précisés, et, d'autre part, notre souche s'en distingue par les points suivants : pigmentation moins intense du mycélium ; vrilles sinistrorses; absence à peu près complète de protéolyse.

\section{RÉSUMÉ}

Nous décrivons les caractères d'une nouvelle espèce de Streptomyces : Streptomyces galtieri n. sp., isolée à partir de lésions spécifiques décelées sur un chien.

$$
* *
$$

Nous tenons à remercier bien vivement $M$. le Professeur Langeron, Directeur de l'Institut de Parasitologie à la Faculté de Médecine de Paris, et l'Equipe d'Etat du Centraal Bureau Voor Schimmelcultures (Baarn, Hollande), Directrice : Mlle J. Westerdijk, pour l'aide précieuse qu'ils nous ont apportée et les conseils qu'ils nous ont prodigués au cours de l'élaboration et de la rédaction de ce travail. Nous leur en demeurons fort reconnaissants (1).

(Laboratoire de Bactériologie de l'Ecole Vétérinaire de Lyon).

(1) Le manuscrit de ce travail était encore en possession du Professeur Langeron qui en corrigeait les épreuves dactylographiées au moment de la disparition de ce Maître dont la compétence était incontestée. Nos brefs remerciements n'expriment qu'imparfaitement toute la reconnaissance que nous devons à sa mémoire. 